\title{
The use of pseudo-inverse methods in reconstructing loads on a missile structure
}

\section{Rajeev Vishwakarma}

School of Engineering and Technology, University of Hertfordshire, Hatfield, Hertfordshire, AL10 9AB, UK

E-mail: R.S.Vishwakarma@herts.ac.uk

\section{Dennis Turner}

Mechanical Analysis Group, MBDA, Stevenage, Hertfordshire, SG1 2DA, UK

E-mail: dennis.turner@mbda-systems.com

\section{Andrew Lewis, Yongkang Chen and Yigeng $\mathrm{Xu}^{*}$}

School of Engineering and Technology, University of Hertfordshire, Hatfield, Hertfordshire, AL10 9AB, UK

E-mail: a.lewis@herts.ac.uk

E-mail: Y.K.Chen@herts.ac.uk

E-mail: y.2.xu@herts.ac.uk

*Corresponding author

\begin{abstract}
A missile during air carriage is subjected to high vibratory forces. These forces consequently impose high levels of stress on the attachment points to the aircraft. A repetitive application of stress causes fatigue. However, since the vibratory forces cannot be measured directly at the interface, an inverse method utilising missile accelerometer data has been used to determine the forces. This paper presents two frequency domain techniques of reconstructing loads. Both techniques utilise the Moore-Penrose pseudo-inverse method, although the second procedure incorporating a normal modes analysis is perhaps better suited for embedding within a health and usage monitoring system (HUMS). Both techniques enable discrete dynamic loads, which are applied to a finite element model in the time domain, to be successfully reconstructed. Consequently, by identifying forces on a missile structure successfully, the presented techniques enable a better structural integrity assessment to be undertaken.
\end{abstract}

Keywords: inverse methods; load reconstruction; aerospace structure; Moore-Penrose pseudo inverse; health and usage monitoring systems; HUMS.

Reference to this paper should be made as follows: Vishwakarma, R., Turner, D., Lewis, A., Chen, Y. and Xu, Y. ( $\mathrm{xxxx})$ 'The use of pseudo-inverse methods in reconstructing loads on a missile structure', Int. J. Modelling, Identification and Control, Vol. X, No. Y, pp.000-000.

Biographical notes: Rajeev Vishwakarma obtained his first class BEng degree in 2008 and Master's degree in 2009 from University of Hertfordshire, School of Engineering and Technology, UK.

Dennis Turner is a Specialist Structural Dynamics, Stress and Vibration Assessment at MBDA. He graduated from The City University in 1974 obtaining his BSc Honours degree in Mechanical Engineering and MSc in Mechanics and Materials in 1979. He has spent approximately 30 years working on various aspects of stress analysis, structural dynamics, vibration data collection, analysis and interpretation in both technical and supervisory roles. He has been involved in a wide variety of projects during his career both at MBDA and elsewhere, including the analysis of composites, metallic airframe structures, modelling distortion in structures during welding and the development of electro-dynamic vibrator test equipment. He holds a patent for the use of novel techniques and materials in vibrator component manufacture and has published several papers on various aspects of structural dynamics and fatigue. He is a member of the Institution of Mechanical Engineers and a Chartered Engineer. 
Andrew Lewis is a Principal Lecturer at the University of Hertfordshire. He graduated from Oxford University in 1979 with an MA in Mathematics; he subsequently obtained his MSc in Applicable Mathematics from the Cranfield Institute of Technology in 1982 and $\mathrm{PhD}$ in Aeronautics from Imperial College, London, in 1994. His career has been divided between the aerospace industry and university sectors. His research interests are in the areas of non-linear aeroelasticity and dynamics, structural dynamics and fluid dynamics.

Yongkang Chen received his BEng and MSc from Taiyuan University of Technology followed by a PhD from the University of Birmingham. Having been a Postdoctoral Research Fellow at the Queen Mary and Westfield College, he was appointed as a Lecturer and then a Principal Lecturer in the School of Engineering and Technology at the University of Hertfordshire. His expertise lies in his interdisciplinary ability to combine linear and non-linear finite element analysis with materials and structural integrity. His research interests include polymer nano-composites, surface engineering including wear, friction and lubrication, energy storage systems, manufacturing modelling and integrity of self-pierce riveting structures.

Yigeng Xu received his BEng and MSc from Zhejiang University of Technology in Mechanical Engineering. He obtained his PhD from the University of Southampton in Engineering Materials. Prior to his appointment as a Senior Lecturer in Aerospace Structure at the University of Hertfordshire, he was a Postdoctoral Research Fellow at Oxford University and an Aerospace Professional Engineer at Airbus UK. His principal research interests have been in the area of fatigue and fracture analysis of lightweight structures, and the development of analytical, numerical and experimental techniques.

\section{Introduction}

Engineering problems can broadly be categorised as a direct or inverse problem. A direct problem is one where the system response can be determined when the system is subjected to a particular input. However, the inverse (or indirect) problem is the opposite, whereby system responses are measured to establish the system inputs (Bekey, 1970; Wang, 2010). Load reconstruction plays a vital part in structural health monitoring/structural integrity assessment (Kankar et al., 2012; Wang et al., 2010), since the identification of real-time flight loads can be used to improve the fatigue assessment. The current study proposes two finite element (FE)-based procedures which incorporate a pseudo-inverse method to reconstruct applied loads.

The Moore-Penrose pseudo-inverse method has been used on several occasions to reconstruct loads. One of the earliest investigations into load reconstruction was undertaken by Giansante et al. (1982), which was based on the work completed by Barlett and Flannelly (1979). The work undertaken by Giansante et al. (1982) involved determining rotor vibratory forces of an AH-1G Helicopter. This was done by using a ground flying test (Giansante et al., 1982) to establish a calibration matrix [which was composed of transfer functions (TF)]. The calibration matrix was used in conjunction with the in-flight acceleration to determine flight loads. These flight loads were applied to the ground flying test rig and the acceleration was measured. The comparison of the predicted responses to the in-flight acceleration produced excellent correlation which indicates successful load reconstruction.

Prior to using the Moore-Penrose method, Okubo et al. (1985) established the impact of noise contamination on the accuracy of force identification, and validated a computer programme via a simple beam structure. The Moore-Penrose method was then used to determine the operating forces acting on three real structures: a machine tool, automobile gas engine, and an air conditioning unit. In all three a known excitation force was applied, and the acceleration response was used to establish the frequency response function's (FRF's). The machines were then run under operating conditions and the acceleration response was used with the FRF's to calculate the operating forces. Since the calculated forces were reasonably identified, the reconstruction can be described as robust.

Hillary and Ewins (1984) also used the methodology with measured strain and acceleration responses to determine forces acting on a cantilever beam. This work was used in a larger investigation to estimate the impact forces on an aircraft engine turbine blade (Hillary, 1983).

The Moore-Penrose pseudo-inverse method was also employed by $\mathrm{Yu}$ and Chan (2003) whilst reconstructing moving loads of two-axle vehicle model in a laboratory experiment using the bridge responses.

The work presented here is a part of a larger investigation. This paper seeks to establish two techniques of reconstructing a known applied force acting on the missile's attachment points (hangers), to the aircraft in the time domain. Both of the frequency domain techniques involves the use of an FE model of a missile structure. By successfully reconstructing forces on a missile structure, the presented methodologies will enable a better structural integrity assessment to be undertaken.

There are six sections to the paper. Section 2 provides some theoretical background to the reconstruction method. The two reconstruction techniques, which utilise the theory, is outlined in Section 3 and applied in Section 4 to a FE representation of a beam model. Section 5 of the paper outlines the results obtained from the two techniques, and general comments are made in the concluding Section 6. 


\section{Theoretical aspect}

The theoretical aspects surrounding the reconstruction Moore-Penrose pseudo-inverse method are outlined in this section. Further details regarding the theoretical aspects are given by Karlsson (1996), Golub and Kahan (1965), and Vishwakarma et al. (2010).

For a discrete linear dynamic system, the equation of motion in the frequency domain (Karlsson, 1996) can be defined as:

$$
\mathrm{Y}(\omega)=\mathrm{H}(\omega) \mathrm{F}(\omega)
$$

where $F(\omega)$ is the excitation force, $H(\omega)$ is the FRF and $\mathrm{Y}(\omega)$ is the response vector.

Consequently, the excitation force can theoretically be determined by rearranging equation (1):

$$
F(\omega)=H(\omega)^{-1} Y(\omega)
$$

The superscript -1 represents the inverse of the matrix. However, this can only be used directly if the FRF matrix is square and non-singular (Stevens, 1987), which implies that the number of unknown forces and responses are equal. If the FRF matrix is rectangular and singular i.e. either overdetermined (when the number of known responses exceeds the number of unknown forces), or under-determined (which has the opposite definition to over-determined), the forces can be estimated through:

$$
\mathrm{F}_{\mathrm{est}}(\omega)=\mathrm{H}^{+}(\omega) \mathrm{Y}(\omega)
$$

where for an over-determined problem:

$$
\mathrm{H}^{+}(\omega)=\left[\mathrm{H}^{\mathrm{T}} \mathrm{H}\right]^{-1} \mathrm{H}^{\mathrm{T}}
$$

And for an under-determined problem:

$$
\mathrm{H}^{+}(\omega)=\mathrm{H}^{\mathrm{T}}\left[\mathrm{H}^{\mathrm{T}} \mathrm{H}\right]^{-1}
$$

Furthermore, $\mathrm{H}^{\mathrm{T}}$ is the Hermitian transpose of $\mathrm{H}$ and superscript + is the pseudo-inverse.

\section{Description of signal preprocessing}

Two reconstruction techniques are outlined in this section. The reconstruction can be used with any kind of responses (accelerations, velocities, displacements or strains - provided the relevant FRF's are also considered).

\subsection{Reconstruction via frequency response analysis}

The reconstruction technique which uses a direct frequency response analysis can be defined through the flow diagram in Figure 1.

The flow diagram in Figure 1 illustrates that the technique utilises acceleration time history and FRF data from the missile structure. A FRF matrix was derived using the direct frequency response analysis in MSC NASTRAN. The elements composing the FRF matrix were determined by applying a unit load to each of the load input positions in turn and thereafter measuring the acceleration response at accelerometer locations ( $\mathrm{x}, \mathrm{y}$, and $\mathrm{z}$ directions) for all frequencies in the range of $0-1025 \mathrm{~Hz}$.

Figure 1 then also exercises the fast Fourier transform (FFT) to convert the acceleration data into the frequency domain. The Moore-Penrose pseudo-inverse method is employed to invert the FRF matrix used in the reconstruction of the applied load.

Figure 1 Reconstruction via frequency response analysis

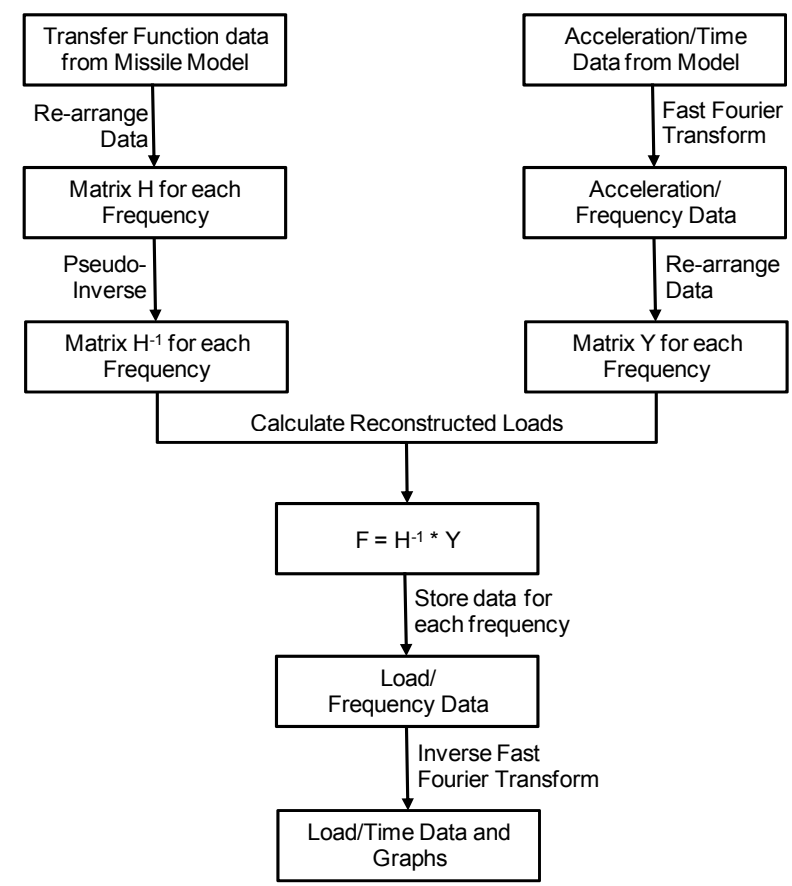

\subsection{Reconstruction via normal mode shapes}

An alternative technique for load reconstruction is shown in the flow chart depicted in Figure 2. The technique employs modal superposition to generate the FRF matrix and involves the use of mass-normalised mode shape vectors.

The first ten natural frequencies and mode shape data of the missile structure were obtained using the Lanczos extraction method in MSC NASTRAN. The missile structure displayed six rigid body motions $(0 \mathrm{~Hz})$ and four elastic modes. In order to account for the rigid body motions the value of $B_{p}$ in (6) was set to 1 for all frequencies.

$$
B_{p}=\frac{(j \omega)^{2}}{\left((j \omega)^{2}+j \omega .2 \varsigma \omega_{n_{p}}+\omega_{n_{p}}{ }^{2}\right)}
$$

Equation (6) was used in parallel with the mode shape displacements for the respective input $(\mathrm{m})$ and response positions (n) (see (7)) to derive the FRF matrix in (8):

$$
\begin{aligned}
& \Phi_{\mathrm{mnp}}^{\prime}=\Phi_{\mathrm{m}_{\mathrm{p}}} \Phi_{\mathrm{n}_{\mathrm{p}}} \\
& \mathrm{H}_{\mathrm{mn}}=\sum_{\mathrm{p}=1}^{10} \mathrm{~B}_{\mathrm{p}} \Phi_{\mathrm{mnp}}^{\prime}
\end{aligned}
$$


As well as using mode-shape data and natural frequencies, the reconstruction process in Figure 2 also utilises acceleration data to reconstruct the initial load curve.

Figure 2 Reconstruction using normal mode shapes

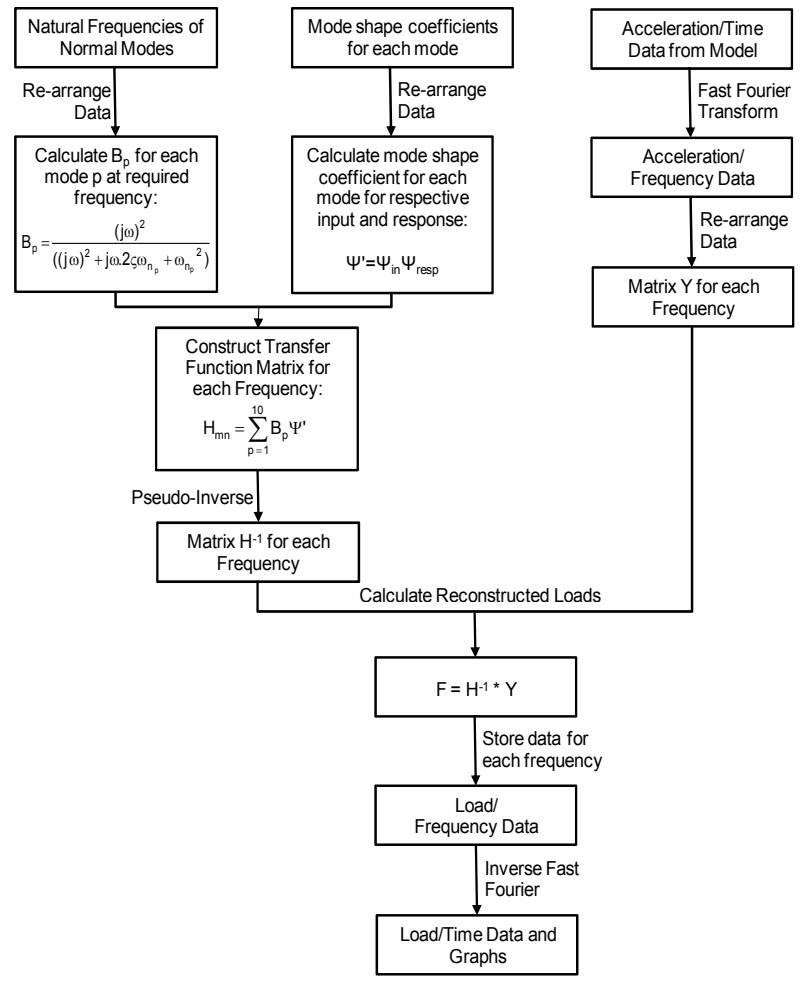

\section{FE missile structure}

The two reconstruction techniques outlined in Section 3 were explored using finite element method (FEM). Prior to undertaking the reconstruction, a FE model of a missile structure was generated in MSC NASTRAN. This section provides details of developing the structure shown in Figure 3.

Figure 3 FEM representation of a missile structure using beam elements

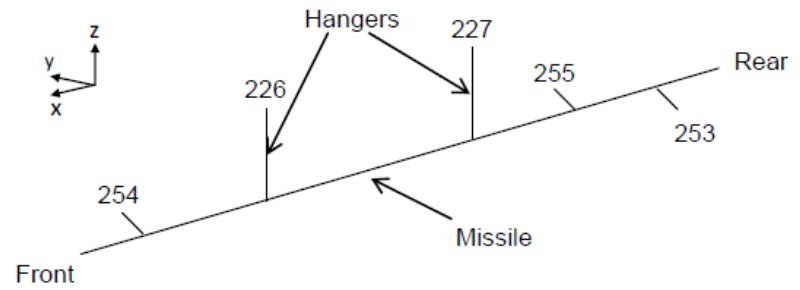

The missile depicted is $1.1 \mathrm{~m}$ long and is composed of $3 \mathrm{D}$ elastic beam (CBAR) elements (MSC NASTRAN User's Guide, 2005). The geometric properties of the missile model are outlined in Table 1.

Also illustrated are the three accelerometer positions at nodes 253, 254 and 255, which are attached to the missile using rigid bar (RBAR) elements (MSC NASTRAN User's Guide, 2005). The missile structure has two $0.05 \mathrm{~m}$ long hangers which are composed of RBAR and scalar spring (CELAS2) elements (MSC NASTRAN User's Guide, 2005). The location of the hangers and accelerometers are summarised in Table 2.

The stiffness's of the CELAS2 elements are summarised in Table 3.

Table 1 Geometric properties of FE missile model

\begin{tabular}{lcc}
\hline Geometric property & Value & Units \\
\hline Radius & 0.025 & $\mathrm{~m}$ \\
X-sectional area & $1.963 \times 10^{-3}$ & $\mathrm{~m}^{2}$ \\
Moment of inertia & $3.067 \times 10^{-7}$ & $\mathrm{~kg} \cdot \mathrm{m}^{2}$ \\
Torsional moment of inertia & $6.134 \times 10^{-7}$ & $\mathrm{~kg} \cdot \mathrm{m}^{2}$ \\
\hline
\end{tabular}

Table 2 Nodal positions of hangers and accelerometers

\begin{tabular}{lcccc}
\hline Structure & Node & $x$ & $y$ & $z$ \\
\hline Accelerometer & 253 & 1.05 & 0.525 & 0.175 \\
Accelerometer & 254 & 0.1 & 0.475 & 0.125 \\
Accelerometer & 255 & 0.75 & 0.475 & 0.15 \\
Forward hanger & 226 & 0.3 & 0.5 & 0.11 \\
Rear hanger & 227 & 0.7 & 0.5 & 0.11 \\
\hline
\end{tabular}

Table 3 Stiffness on Fwd and Aft hangers

\begin{tabular}{lccc}
\hline Position & Element & $\begin{array}{c}\text { Stiffness } \\
(\mathrm{N} / \mathrm{m})\end{array}$ & $\begin{array}{c}\text { Degrees of } \\
\text { freedom }\end{array}$ \\
\hline Fwd hanger & 226 & $10^{8}$ & $\mathrm{x}$ \\
Fwd hanger & 226 & $10^{8}$ & $\mathrm{y}$ \\
Fwd hanger & 226 & $10^{8}$ & $\mathrm{z}$ \\
Aft hanger & 227 & $10^{8}$ & $\mathrm{x}$ \\
Aft hanger & 227 & $10^{8}$ & $\mathrm{y}$ \\
Aft hanger & 227 & $10^{8}$ & $\mathrm{z}$ \\
Aft hanger & 227 & $10^{6 *}$ & $\mathrm{Rx}$ \\
\hline
\end{tabular}

Note: *The unit for this stiffness is $\mathrm{N}-\mathrm{m} / \mathrm{rad}$.

The missile mass representations include point masses of $0.8 \mathrm{~kg}$ placed at the missile node positions and a non-structural mass of $20 \mathrm{~kg} / \mathrm{m}$. The point masses have been modelled using CONM2 elements (MSC NASTRAN User's Guide, 2005).

The structure is homogeneous and is constructed using an aluminium alloy. The material properties of the aluminium alloy can be summarised in Table 4 .

Table 4 Nodal positions of hangers and accelerometers

\begin{tabular}{lc}
\hline Parameter & Quantity \\
\hline Young's modulus & $70 \mathrm{GPa}$ \\
Poisson's ratio & 0.32 \\
Density & $2,730 \mathrm{~kg} / \mathrm{m}^{3}$ \\
\hline
\end{tabular}

A constant modal damping ratio of 0.02 has been used throughout. 
The resulting FE model was subjected to the two impulse loading conditions. The loading conditions were modelled using TABLED1 card in MSC NASTRAN.

\subsection{Step load condition 1}

This load condition is depicted in Figure 4.

Figure 4 Applied step load condition 1

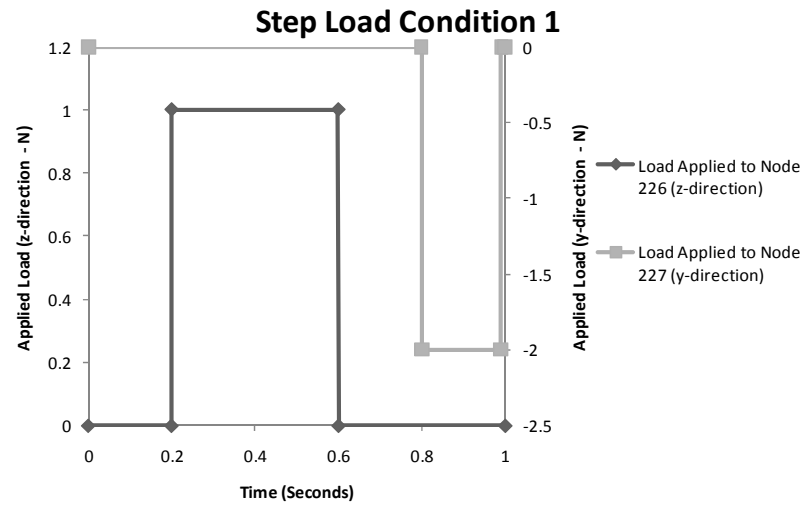

The diagram in Figure 4 depicts a unit load applied as an impulse on node 226 (z-direction) of the structure in Figure 3, Furthermore, after a break of 0.200568 seconds, a $-2 \mathrm{~N}$ load was applied to the structure at node 227 (y-direction). Figure 4 outlines that the load on node 226 was applied for duration of 0.399184 seconds, and the load applied on node 227 was for duration of 0.189832 seconds.

\subsection{Step load condition 2}

This load condition is depicted in Figure 5.

The diagram in Figure 5 depicts a unit load applied as an impulse on nodes 226 and 227 (y-direction) of the structure in Figure 3, Furthermore, a $-2 \mathrm{~N}$ load was applied to the structure at node 226 and 227 (z-direction). Each of the impulses outlined Figure 5 was applied to the structure for 0.12 seconds.

Figure 5 Applied step load condition 2

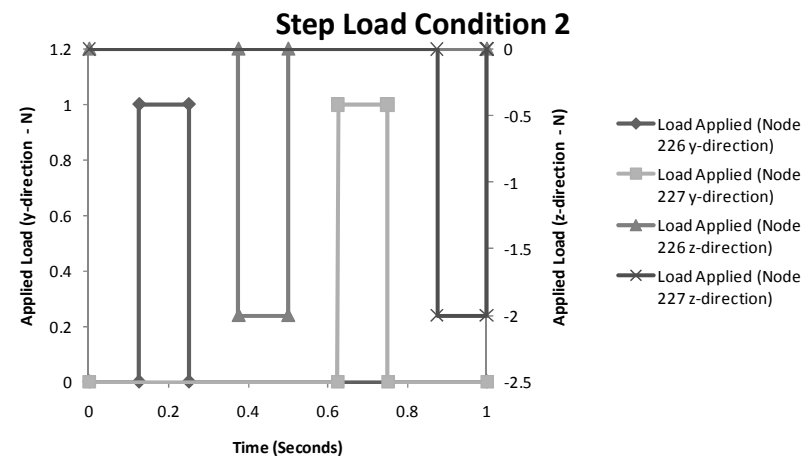

\section{Results}

Figure 6 and Figure 7 outlines some of the results which have been predicted using the two techniques depicted in Figures 1 and 2, when the FE missile model is subjected to the load profiles depicted in Figure 4 and Figure 5.

Figure 6 Step load condition 1: reconstructed loads, (a) point 226 reconstructed forces via frequency response analysis (b) point 226 reconstructed forces via normal mode shapes (c) point 227 reconstructed forces via frequency response analysis (d) point 227 reconstructed forces via normal mode shapes (see online version for colours)

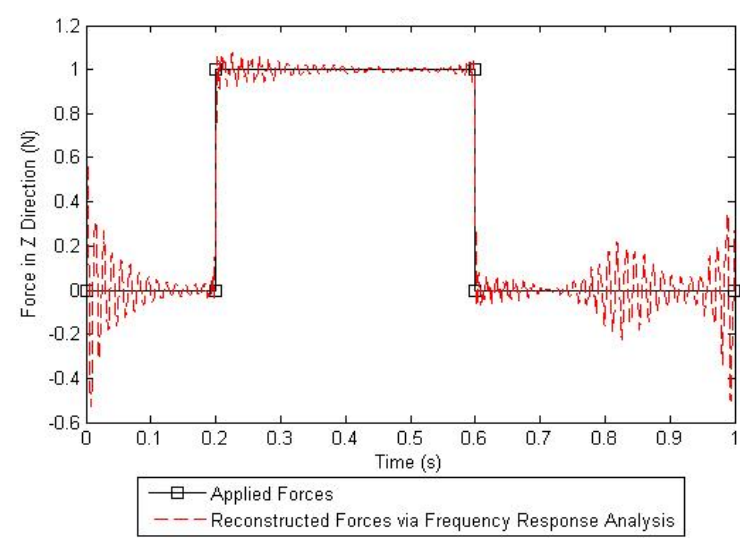

(a)

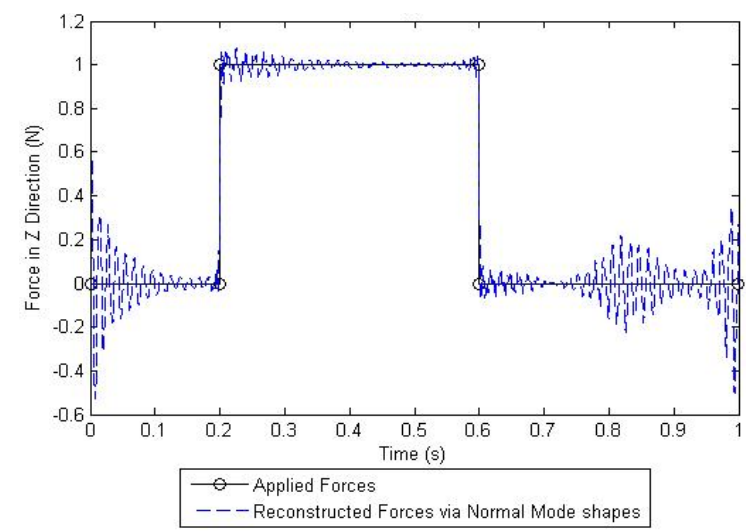

(b)

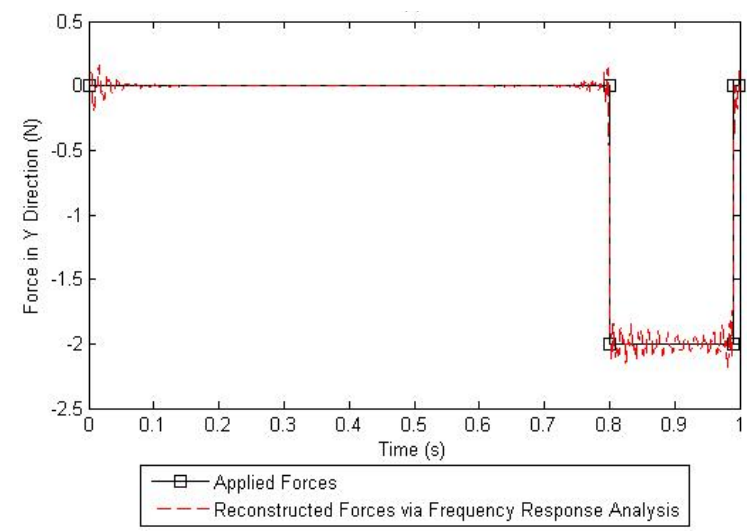

(c) 
Figure 6 Step load condition 1: reconstructed loads, (a) point 226 reconstructed forces via frequency response analysis (b) point 226 reconstructed forces via normal mode shapes (c) point 227 reconstructed forces via frequency response analysis (d) point 227 reconstructed forces via normal mode shapes (continued) (see online version for colours)

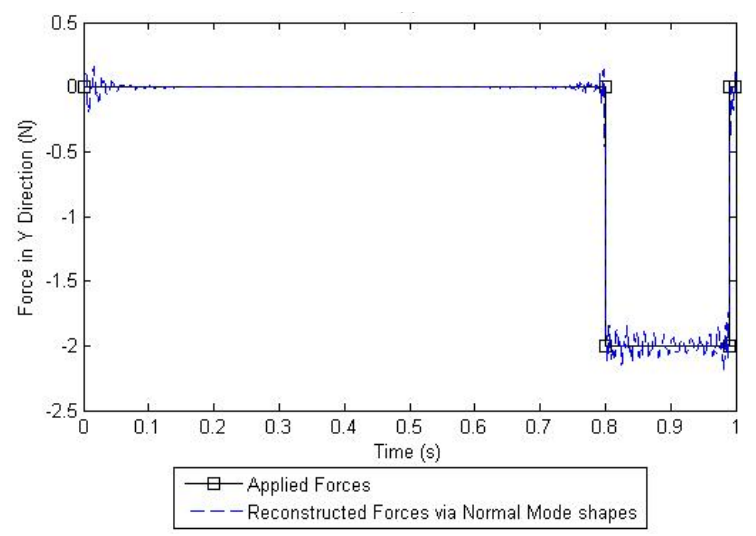

(d)

The correlation coefficient $\left(\rho_{\mathrm{xy}}\right)$ and normalised root mean square error (NRMSE) between the applied and reconstructed loads, for step load condition 1, are indicated in Table 5:

Table 5 Correlation coefficients and NRMSE for step load reconstruction

\begin{tabular}{lcclcc}
\hline \multirow{2}{*}{$\begin{array}{l}\text { Reconstruction } \\
\text { technique }\end{array}$} & \multicolumn{2}{c}{226 (z direction) } & & \multicolumn{2}{c}{227 (y direction) } \\
\cline { 2 - 3 } \cline { 5 - 6 } & $\rho_{x y}$ & NRMSE & & $\rho_{x y}$ & NRMSE \\
\hline $\begin{array}{l}\text { Via frequency } \\
\text { response analysis }\end{array}$ & 0.98 & 0.05 & & 0.98 & 0.05 \\
$\begin{array}{l}\text { Via normal mode } \\
\text { shapes }\end{array}$ & 0.98 & 0.05 & & 0.98 & 0.05 \\
\hline
\end{tabular}

The correlation coefficient $\left(\rho_{\mathrm{xy}}\right)$ and normalised root mean square error (NRMSE) between the applied and reconstructed loads, for step load condition 2, are indicated in Table 6:

Table 6 Correlation coefficients and NRMSE for step load reconstruction

\begin{tabular}{|c|c|c|c|c|}
\hline \multirow{2}{*}{$\begin{array}{l}\text { Reconstruction } \\
\text { technique }\end{array}$} & \multicolumn{2}{|c|}{226 (y direction) } & \multicolumn{2}{|c|}{226 ( $z$ direction $)$} \\
\hline & $\rho_{x y}$ & NRMSE & $\rho_{x y}$ & NRMSE \\
\hline $\begin{array}{l}\text { Via frequency } \\
\text { response analysis }\end{array}$ & 0.99 & 0.03 & 0.99 & 0.03 \\
\hline \multirow{3}{*}{$\begin{array}{l}\text { Via normal mode } \\
\text { shapes }\end{array}$} & 0.99 & 0.03 & 0.99 & 0.03 \\
\hline & \multicolumn{2}{|c|}{227 (y direction) } & \multicolumn{2}{|c|}{227 ( $z$ direction) } \\
\hline & $\rho_{x y}$ & NRMSE & $\rho_{x y}$ & NRMSE \\
\hline $\begin{array}{l}\text { Via frequency } \\
\text { response analysis }\end{array}$ & 0.99 & 0.03 & 0.99 & 0.03 \\
\hline $\begin{array}{l}\text { Via normal mode } \\
\text { shapes }\end{array}$ & 0.99 & 0.03 & 0.99 & 0.03 \\
\hline
\end{tabular}

The results depicted in Figures 6 and 7 and Tables 5 and 6 indicates a successful prediction of the applied loads outlined in Section 3. The results also indicate that the loads predicted by the two pseudo-inverse techniques are similar.

Figure 7 Step load condition 2: reconstructed loads, (a) point 226 reconstructed forces via frequency response analysis (Y direction) (b) point 226 reconstructed forces via normal mode shapes (Y direction) (c) point 226 reconstructed forces via frequency response analysis ( $\mathrm{Z}$ direction) (d) point 226 reconstructed forces via normal mode shapes ( $Z$ direction) (e) point 227 reconstructed forces via frequency response analysis (Y direction) (f) point 227 reconstructed forces via normal mode shapes (Y direction) (g) point 227 reconstructed forces via frequency response analysis ( $\mathrm{Z}$ direction) (h) point 227 reconstructed forces via normal mode shapes ( $\mathrm{Z}$ direction) (see online version for colours)

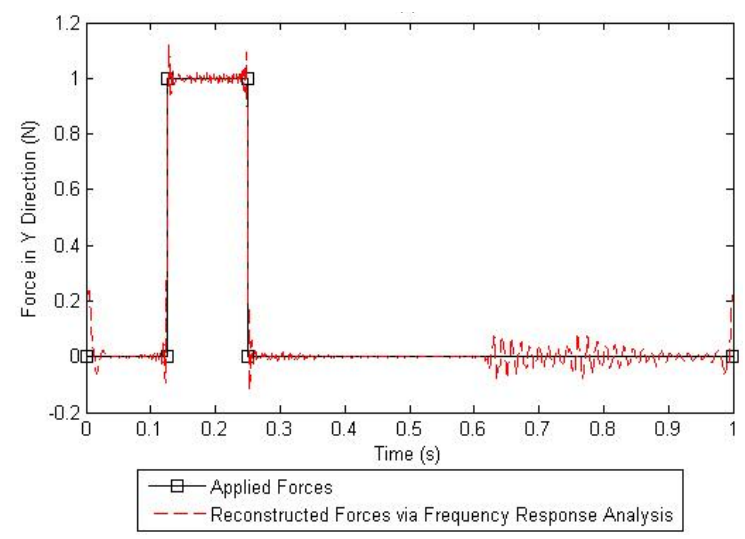

(a)

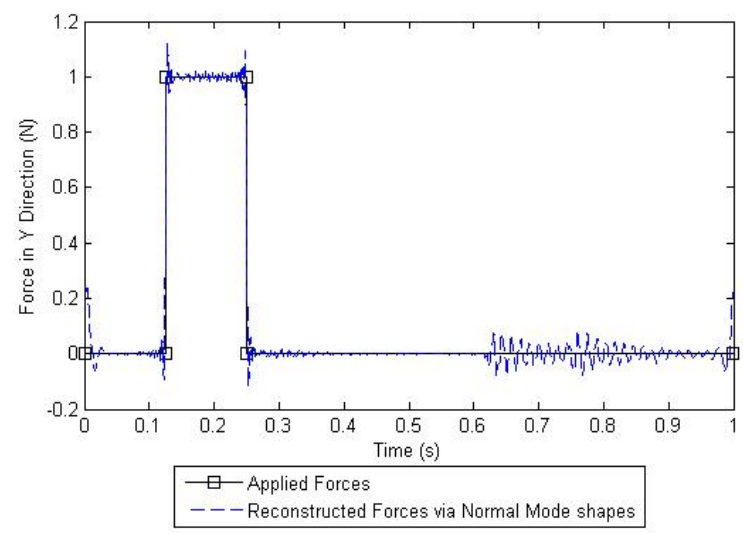

(b) 
Figure 7 Step load condition 2: reconstructed loads, (a) point 226 reconstructed forces via frequency response analysis (Y direction) (b) point 226 reconstructed forces via normal mode shapes (Y direction) (c) point 226 reconstructed forces via frequency response analysis ( $Z$ direction) $(d)$ point 226 reconstructed forces via normal mode shapes ( $Z$ direction) (e) point 227 reconstructed forces via frequency response analysis (Y direction) (f) point 227 reconstructed forces via normal mode shapes (Y direction) $(\mathrm{g}$ ) point 227 reconstructed forces via frequency response analysis (Z direction) (h) point 227 reconstructed forces via normal mode shapes ( $\mathrm{Z}$ direction) (continued) (see online version for colours)

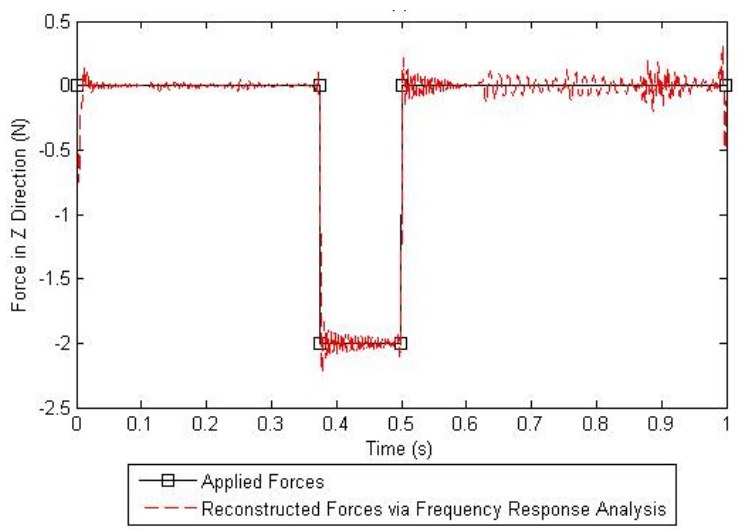

(c)

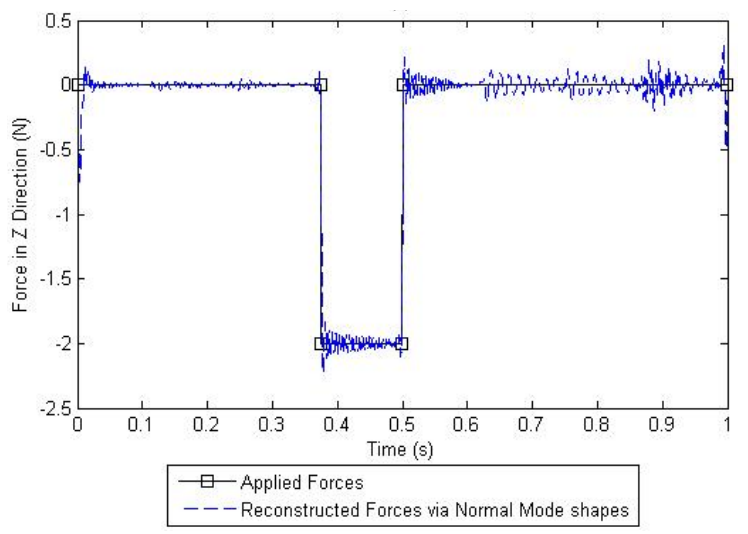

(d)

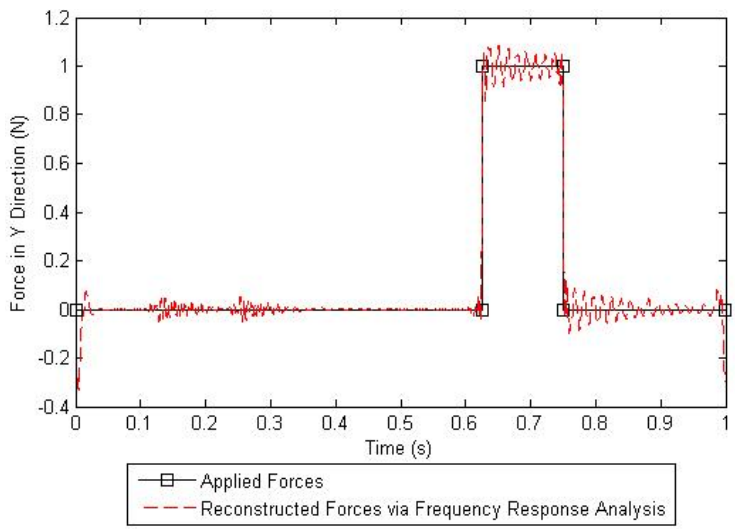

(e)
Figure 7 Step load condition 2: reconstructed loads, (a) point 226 reconstructed forces via frequency response analysis (Y direction) (b) point 226 reconstructed forces via normal mode shapes (Y direction) (c) point 226 reconstructed forces via frequency response analysis ( $Z$ direction) (d) point 226 reconstructed forces via normal mode shapes ( $Z$ direction) (e) point 227 reconstructed forces via frequency response analysis (Y direction) (f) point 227 reconstructed forces via normal mode shapes (Y direction) (g) point 227 reconstructed forces via frequency response analysis ( $\mathrm{Z}$ direction) (h) point 227 reconstructed forces via normal mode shapes ( $\mathrm{Z}$ direction) (continued) (see online version for colours)

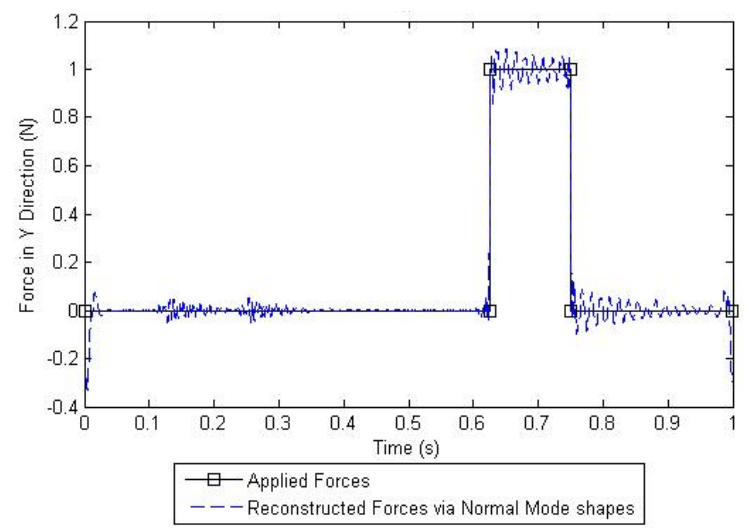

(f)

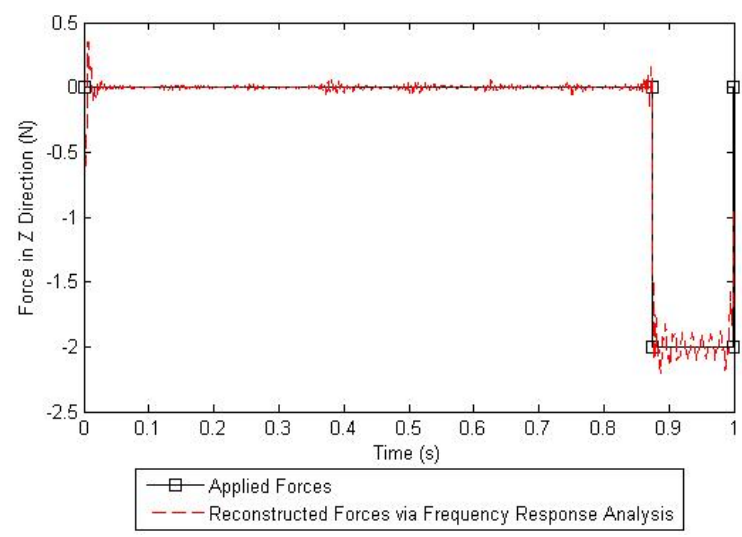

(g)

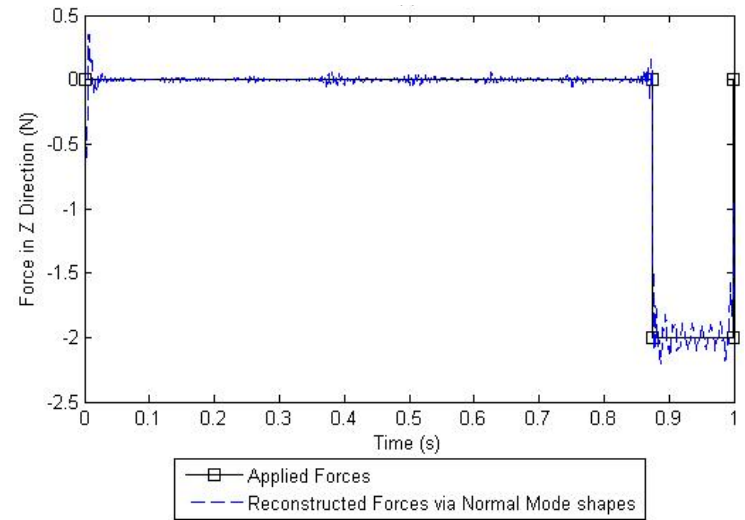

(h) 
The results obtained from the two reconstruction techniques are similar and demonstrates that the two loading cases have been predicted successfully. Figures 6 and 7 depicts that the forces predicted by the two methods have a similar magnitude and frequency in comparison to the applied load curves. The accuracy of the reconstruction is highlighted in Table 5 and Table 6 through the correlation coefficients, which is greater than 0.9 , which further supports the success of the reconstruction techniques.

The predicted forces from the two reconstruction techniques do consist of some discrepancies. Figure 6 and Figure 7 indicates that the two techniques reconstruct an overshoot at the particular instants in time whereby the impulse force has been applied and removed from the structure. The presence of the overshoot has occurred due to Gibbs phenomenon, when a point of discontinuity is analysed with the FFT process (or iFFT process) (Kammler, 2000; Kreyszig, 1999).

The two techniques are very consistent in terms of the reconstructed loads, since the correlation coefficient is similar. The consistency of the two techniques is also highlighted through the calculation of the NRMSE. The NRMSE is the same for each reconstruction technique which would suggest that the two techniques are as successful as each other.

From an experimental perspective, it can be suggested that the calculation of the FRF's from both techniques will be inaccurate due of noise. Whilst calculating the FRF's, noise will occur due to the size of the frequency increment whilst using a swept sinusoidal wave. Consequently, a smaller frequency increment may incur larger noise between adjacent frequencies. Whilst using the normalised mode shapes, the measurement of the mode shape data influences the accuracy of the FRF's.

With respect to efficiency, it can be suggested that the use of normalised mode shape vectors (via modal super-position analysis) is better than using the direct frequency response analysis. The use of normalised mode-shape vectors provides compact storage and improves computation time and effort as the FRF matrix can be calculated as required.

Experimental work has been undertaken to validate the two reconstruction methods (depicted in Section 3), and has been documented in Vishwakarma (2012).

\section{Concluding remarks}

1 Load reconstruction is a useful tool to determine the magnitude of the excitation initially applied to the structure. Two techniques have been employed to successfully predict the loads at the interface of the missile and the wing.

2 The two frequency domain techniques were examined whilst reconstructing two loading conditions applied to a FE missile model.
3 The investigation indicated that the Moore-Penrose pseudo-inverse technique can provide good estimations of applied forces.

4 With respect to composing the FRF matrices, two approaches were investigated. One of the approaches utilises a frequency response analysis whilst the other utilises mass-normalised mode shape vectors (via modal super-position). It is evident that the later approach provides extremely compact storage and allows the FRF matrix to be calculated as required. Consequently, the technique which uses mass-normalised mode shapes maybe suitable for use within a health and usage monitoring system (HUMS) to identify forces more efficiently.

\section{Acknowledgements}

The research was financially supported by MBDA and EPSRC.

\section{References}

Barlett, F. and Flannelly, W.G. (1979) 'Modal verification of force determination for measuring vibratory loads', Journal of the American Helicopter Society, Vol. 19, No. 4, pp.10-18.

Bekey, G. (1970) 'System identification - an introduction and a survey', Simulation, Vol. 15, No. 4, pp.151-166.

Giansante, N., Jones, R. and Galapodas, N. (1982) 'Determination of in-flight helicopter loads', Journal of the American Helicopter Society, Vol. 27, No. 3, pp.58-64.

Golub, G. and Kahan, W. (1965) 'Calculating the singular values and pseudo-inverse of a matrix', Journal of the Society for Industrial and Applied Mathematics: Series B, Numerical Analysis, Vol. 2, No. 2, pp.205-224.

Hillary, B. (1983) Indirect Measurement of Vibration Excitation Sources, Imperial College of Science and Technology, UK.

Hillary, B. and Ewins, D.J. (1984) 'The use of strain gages in force determination and frequency response measurements', Proc. 2nd International Modal Analysis Conf., Orlando, FL, pp.627-634.

Kammler, D. (2000) A First Course in Fourier Analysis, Prentice-Hall, Upper Saddle River, New Jersey.

Kankar, P., Sharma, S. and Harsha, S. (2012) 'Vibration-based fault diagnosis of a rotor bearing system using artificial neural network and support vector machine', International Journal of Modelling Identification and Control, Vol. 15, No. 3, pp.185-198.

Karlsson, S. (1996) 'Identification of external structural loads from measured harmonic responses', Journal of Sound and Vibration, Vol. 196, No. 1, pp.59-74.

Kreyszig, E. (1999) Advanced Engineering Mathematics, 8th ed., John Wiley and Sons Inc., New Jersey, USA.

MSC NASTRAN User's Guide (2005) MSC.Software Corporation, USA.

Okubo, N., Tanabe, S. and Tatsunso, T. (1985) 'Identification of forces generated by a machine under operation condition', Proc. of 3rd Int. Modal Analysis, USA, pp.920-927.

Stevens, K. (1987) 'Force identification problems - an overview', Proc. SEM Spring Meeting, Boca Raton, Florida, pp.838-844. 
Vishwakarma, R. (2012) 'The use of inverse methods in determining applied loads to aerospace structure', $\mathrm{PhD}$ thesis, University of Hertfordshire, UK.

Vishwakarma, R., Turner, D., Lewis, A., Chen, Y., Houghton, P. and $\mathrm{Xu}, \mathrm{Y}$. (2010) 'Load reconstruction on a missile structure using the pseudo-inverse methods', International Conference on Modelling, Identification and Control, Okayama, pp.100-105.

Wang, S. (2010) 'Tomography in frequency domain using wave equation', International Journal of Modelling Identification and Control, Vol. 9, No. 3, pp.262-266

Wang, Y., Xu, X. and Cai, W. (2010) 'Online multivariable process identification in the frequency domain', International Journal of Modelling Identification and Control, Vol. 10, No. 1, pp.106-111.

$\mathrm{Yu}, \mathrm{L}$. and Chan, H. (2003) 'Moving force identification based on the frequency-time domain method', Journal of Sound and Vibration, Vol. 261, No. 2, pp.329-349. 